\title{
Positive Solutions for Discrete Boundary Value Problems to One-Dimensional $p$-Laplacian with Delay
}

\author{
Linjun Wang and Xumei Chen \\ Faculty of Science, Jiangsu University, Zhenjiang 212013, China \\ Correspondence should be addressed to Xumei Chen; chenxumei@ujs.edu.cn
}

Received 23 April 2013; Accepted 20 June 2013

Academic Editor: Junjie Wei

Copyright (C) 2013 L. Wang and X. Chen. This is an open access article distributed under the Creative Commons Attribution License, which permits unrestricted use, distribution, and reproduction in any medium, provided the original work is properly cited.

We study the existence of positive solutions for discrete boundary value problems to one-dimensional $p$-Laplacian with delay. The proof is based on the Guo-Krasnoselskii fixed-point theorem in cones. Two numerical examples are also provided to illustrate the theoretical results.

\section{Introduction}

The $p$-Laplacian differential equations have been vastly applied in many fields such as non-Newtonian mechanics, economics, ecology, neural networks, and nonlinear flow laws, [1-5].

One of the important examples was described in [6]. Let $x=\left(x_{1}, x_{2}\right)$ be the two Cartesian coordinates in the plane of the glacier occupying the Lipschitzian domain $\Omega$, we denote by $u(x)$ the horizontal velocity component of the ice at the point $x$. After a rescaling of the physical velocity of the ice, $u$ satisfies the following equation:

$$
-\operatorname{div}(\psi(|\nabla u|) \nabla u)=e, \quad \text { in } \Omega,
$$

where $e$ is a hydrostatic pressure force acting on the glacier and $\psi$ is a function resulting from a constitutive law for the ice. The typical case of (1) is the following equation:

$$
\operatorname{div}\left(\phi_{p}(\nabla u)\right)+b(|x|) g(u)=0, \quad x \in \frac{B\left(0, R_{2}\right)}{B\left(0, R_{1}\right)},
$$

where $\phi_{p}(s)=|s|^{p-2} s$ with $p>1$ and $B\left(0, R_{i}\right) \subset \mathbb{R}^{d}$ are the open balls centred about the origin with radius $R_{i}$, respectively. People are interested to consider the positive radial solutions of (2), and then (2) can be reduced to the following form [7]:

$$
r^{1-d}\left(r^{d-1} \phi_{p}\left(u^{\prime}\right)\right)^{\prime}+b(r) g(u(r))=0, \quad R_{1}<r<R_{2} .
$$

Let $s=-\int_{r}^{R_{2}} t^{-(d-1) /(p-1)} d t, v(s)=u(r(s))$, and $\rho=$ $-\int_{R_{1}}^{R_{2}} t^{-(d-1) /(p-1)} d t$. Then (3) is transformed to

$$
\begin{array}{r}
\left(\phi_{p}\left(v^{\prime}(s)\right)\right)^{\prime}+r^{p(d-1) /(p-1)}(s) b(r(s)) g(v(s))=0, \\
\rho<s<0 .
\end{array}
$$

With variable change $t=1-s / \rho$ and $y(t)=v(s)$, (4) reads

$$
\left(\phi_{p}\left(y^{\prime}(t)\right)\right)^{\prime}+c(t) g(y(t))=0, \quad 0<t<1,
$$

where $c(t)=(-\rho)^{p} r^{(p(d-1)) /(p-1)}[\rho(1-t)] b[r(\rho(1-t))]$. Equation (5) is a typical type of one-dimensional $p$-Laplacian equation.

In the real world, some processes are more reasonably described as $p$-Laplacian differential equations with delay $[2,8,9]$. The reason is that the differential of the unknown solutions depends not only on the values of the unknown solutions at the current time but also on the values prior to that. Such equations, to a certain extent, reflect much more exactly the physical reality than the equations without delay.

In reality, (5) is applied together with some boundary value conditions, see for example, $[1,4,8-11]$. By using the Guo-Krasnoselskii fixed-point theorem, Jin and Yin [9] 
proved the existence of one positive solutions for the following boundary value problem of one-dimensional $p$-Laplacian with delay

$$
\begin{gathered}
\left(\phi_{p}\left(u^{\prime}(t)\right)\right)^{\prime}+\lambda g(t, u(t-\tau))=0, \quad 0<t<1, \tau>0, \\
u(1)=0, \quad u(t)=0, \quad-\tau \leq t \leq 0 .
\end{gathered}
$$

Based on a fixed-point approach, Bai and Xu [8] obtained the existence of positive solutions for problem (6) with $g(t, u)$ having some singularities.

The main motivation of our work is twofold. The one is to prove the existence of positive solutions for discrete boundary value problems with delay. It is of interest to note here that the existence of single and multiple positive solutions for discrete boundary value problems to one-dimensional $p$-Laplacian have been studied in great detail in the literature [12-18] and the references therein. However, there are few papers dealing with the existence of positive solutions for discrete boundary value problems to one-dimensional $p$-Laplacian with delay.

The other motivation is coming from the numerical solutions of problem (6). In order to get the numerical solutions of problem (6), we can apply the standard Euler method to discretize problem (6) and approximate its solutions numerically. An immediate and natural question arises if the corresponding difference equation together with boundary conditions has positive solutions.

Motivated by above, our purpose in this paper is to show the existence of positive solutions for the discretization equations of problem (6). Namely, we will prove the existence of positive solutions of the following problem:

$$
\begin{gathered}
\Delta\left(\phi_{p}(\Delta u(n-1))\right)+\lambda a(n) f\left(n, u\left(n-n_{0}\right)\right)=0, \\
n \in\{1,2, \ldots, N\}, \\
u(N+1)=0, \quad u(n)=0, \quad n \in\left\{-n_{0},-n_{0}+1, \ldots, 0\right\},
\end{gathered}
$$

where $\Delta u(n)=u(n+1)-u(n)$ and $n_{0}$ is a positive integer.

This paper is organized as follows. In Section 2, we introduce some basic definitions and then we state the GuoKrasnoselskii fixed-point theorem. In Section 3, we write a representation for a solution to problem (7) in terms of the fixed point of an appropriate operator. Then we prove that problem (7) has a positive solution with $\lambda$ belonging to an open interval by employing the fixed-point theorem. In Section 4, two numerical examples are presented to illustrate the theoretical results.

\section{Preliminaries}

In this section, we introduce some basic definitions and recall the Guo-Krasnoselskii fixed-point theorem which plays a fundamental role in our subsequent analysis.

For convenience, we will let $\mathbf{Z}(a, b)$ denote $\{a, a+1, \ldots$, $b\}$, where $a<b \in \mathbb{Z}$.

A sequence $(u(n))_{n=-n_{0}}^{N+1}$ is said to be a positive solution of (7), if it satisfies (7) with $u(n)>0, n \in \mathbf{Z}(1, N+1)$.

We collect some properties of the function $\phi_{p}(\cdot)$.
Lemma 1. $\phi_{p}(s)$ is increasing in $s$ and $\left(\phi_{p}\right)^{-1}=\phi_{q}$, with $1 / p+$ $1 / q=1 ; \phi_{p}\left(s_{1} \cdot s_{2}\right)=\phi_{p}\left(s_{1}\right) \cdot \phi_{p}\left(s_{2}\right)$.

Definition 2. Let $E$ be a real Banach space. A nonempty closed convex set $K \subset E$ is called a cone if

(i) $x \in K$ and $\lambda \geq 0$ imply $\lambda x \in K$,

(ii) $x \in K$ and $-x \in P$ imply $x=\theta$.

Theorem 3 (see [19]). Let $E$ be a Banach space and $K \subset E$ a cone. Assume $\Omega_{1}, \Omega_{2}$ are bounded open subsets of $E$ with $\theta \in$ $\Omega_{1}, \bar{\Omega}_{1} \subset \Omega_{2}$, and let $T: K \bigcap\left(\bar{\Omega}_{2} \backslash \Omega_{1}\right) \rightarrow K$ be a completely continuous operator such that one of the following holds:

(i) $\|T u\| \leq\|u\|, u \in K \cap \partial \Omega_{1},\|T u\| \geq\|u\|, u \in$ $K \cap \partial \Omega_{2}$;

(ii) $\|T u\| \geq\|u\|, u \in K \cap \partial \Omega_{1},\|T u\| \leq\|u\|, u \in K \cap \partial \Omega_{2}$.

Then $T$ has a fixed point in $K \cap\left(\bar{\Omega}_{2} \backslash \Omega_{1}\right)$.

\section{Existence of Positive Solutions}

In this section, we study the existence of positive solutions for the following equation with delay:

$$
\begin{array}{r}
\Delta\left(\phi_{p}(\Delta u(n-1))\right)+\lambda a(n) f\left(n, u\left(n-n_{0}\right)\right)=0, \\
n \in \mathbf{Z}(1, N),
\end{array}
$$

subject to the boundary condition

$$
u(N+1)=0, \quad u(n)=0, \quad n \in \mathbf{Z}\left(-n_{0}, 0\right) .
$$

We assume that

(A1) $f: \mathbf{Z}(1, N+1) \times \mathbb{R} \rightarrow \mathbb{R}^{+}$is a continuous function $\left(\mathbb{R}^{+}\right.$denotes the set of nonnegative reals);

(A2) $a(\cdot)$ is a positive function defined on $\mathbf{Z}(1, N+1)$, $\sum_{i=1}^{N+1} a(i)<\infty$.

Remark 4. Recall that a map $f: \mathbf{Z}(1, N+1) \times \mathbb{R} \rightarrow \mathbb{R}^{+}$is continuous if it is continuous as a map of the topological space $\mathbf{Z}(1, N+1) \times \mathbb{R}$ into the topological space $\mathbb{R}^{+}$. Throughout this paper, the topology on $\mathbf{Z}(1, N+1)$ will be the discrete topology.

Rewrite (8) as

$$
\Delta\left(\phi_{p}(\Delta u(i-1))\right)=-\lambda a(i) f\left(i, u\left(i-n_{0}\right)\right) .
$$

Summing up (10) with respect to $i$ from $m$ to $N$ leads to

$$
\phi_{p}(\Delta u(m-1))-\phi_{p}(\Delta u(0))=-\sum_{i=1}^{m-1} \lambda a(i) f\left(i, u\left(i-n_{0}\right)\right) \text {. }
$$

Noticing Lemma 1, we have

$$
\Delta u(m-1)=\phi_{q}\left(\phi_{p}(\Delta u(0))-\sum_{i=1}^{m-1} \lambda a(i) f\left(i, u\left(i-n_{0}\right)\right)\right) .
$$


Summing up (12) with respect to $m$ from 1 to $n$ and noticing the boundary condition (9) lead to

$$
u(n)=\sum_{m=1}^{n} \phi_{q}\left(\phi_{p}(\Delta u(0))-\sum_{i=1}^{m-1} \lambda a(i) f\left(i, u\left(i-n_{0}\right)\right)\right),
$$

where $\Delta u(0)$ satisfies

$$
F(x)=\sum_{m=1}^{N+1} \phi_{q}\left(\phi_{p}(x)-\sum_{i=1}^{m-1} \lambda a(i) f\left(i, u\left(i-n_{0}\right)\right)\right)=0 .
$$

Obviously, $F(x)$ is continuous and strictly increasing. We have

$$
F(0)<0, \quad F\left(\phi_{q}\left(\sum_{i=1}^{N+1} \lambda a(i) f\left(i, u\left(i-n_{0}\right)\right)\right)\right)>0 .
$$

Thus, there exists a unique solution to (14)

$$
\xi_{u} \in\left(0, \phi_{q}\left(\sum_{i=1}^{N+1} \lambda a(i) f\left(i, u\left(i-n_{0}\right)\right)\right)\right) .
$$

Let

$$
g(n)=\phi_{q}\left(\sum_{i=1}^{n} \lambda a(i) f\left(i, u\left(i-n_{0}\right)\right)\right) .
$$

Since $g(n)$ is increasing and $g(0)=0$, we have $\xi_{u} \in$ $(g(0), g(N+1))$. There exists $n_{1} \in \mathbf{Z}(0, N)$ that satisfied

$$
\begin{aligned}
& \phi_{q}\left(\sum_{i=1}^{n_{1}} \lambda a(i) f\left(i, u\left(i-n_{0}\right)\right)\right) \\
& \quad \leq \xi_{u}<\phi_{q}\left(\sum_{i=1}^{n_{1}+1} \lambda a(i) f\left(i, u\left(i-n_{0}\right)\right)\right) .
\end{aligned}
$$

Hence,

$$
\xi_{u}=\phi_{q}\left(\sum_{i=1}^{n_{1}} \lambda a(i) f\left(i, u\left(i-n_{0}\right)\right)+\omega\right),
$$

where $\omega \in\left[0, \lambda a\left(n_{1}+1\right) f\left(n_{1}+1, u\left(n_{1}+1-n_{0}\right)\right)\right)$.

By (13) and (19), together with the definition of the positive solution, it is easy to get

$$
u(n)=\sum_{m=1}^{n} \phi_{q}\left(\sum_{i=m}^{n_{1}} \lambda a(i) f\left(i, u\left(i-n_{0}\right)\right)+\omega\right), \quad n<n_{1}+1 .
$$

Similarly, summing up (12) with respect to $m$ from $n+1$ to $N+1$ and noticing (9), we have

$$
\begin{aligned}
& u(n)=-\sum_{m=n+1}^{N+1} \phi_{q} \\
& \phi_{p}(\Delta u(0)) \\
&\left.-\sum_{i=1}^{m-1} \lambda a(i) f\left(i, u\left(i-n_{0}\right)\right)\right), \\
& n>n_{1}-1 .
\end{aligned}
$$

Then by substituting (19) in (21), we obtain

$$
\begin{array}{r}
u(n)=\sum_{m=n+1}^{N+1} \phi_{q}\left(\sum_{i=n_{1}+1}^{m-1} \lambda a(i) f\left(i, u\left(i-n_{0}\right)\right)-\omega\right), \\
n>n_{1}-1 .
\end{array}
$$

Since (19) is the solution of (14), we have

$$
\begin{gathered}
F(x)=\sum_{m=1}^{N+1} \phi_{q}\left(\phi_{p}\left(\phi_{q}\left(\sum_{i=1}^{n_{1}} \lambda a(i) f\left(i, u\left(i-n_{0}\right)\right)+\omega\right)\right)\right. \\
\left.-\sum_{i=1}^{m-1} \lambda a(i) f\left(i, u\left(i-n_{0}\right)\right)\right)=0 .
\end{gathered}
$$

Namely,

$$
\begin{aligned}
\sum_{m=1}^{N+1} \phi_{q}( & \left(\sum_{i=1}^{n_{1}} \lambda a(i) f\left(i, u\left(i-n_{0}\right)\right)+\omega\right) \\
- & \left.\sum_{i=1}^{m-1} \lambda a(i) f\left(i, u\left(i-n_{0}\right)\right)\right)=0 .
\end{aligned}
$$

Thus,

$$
\begin{aligned}
\sum_{m=1}^{n_{1}} \phi_{q} & \left(\sum_{i=m}^{n_{1}} \lambda a(i) f\left(i, u\left(i-n_{0}\right)\right)+\omega\right) \\
& =\sum_{m=n_{1}+1}^{N+1} \phi_{q}\left(\sum_{i=n_{1}+1}^{m-1} \lambda a(i) f\left(i, u\left(i-n_{0}\right)\right)-\omega\right) .
\end{aligned}
$$

Therefore, if $u(n)$ is a positive solution of the boundary value problems (8) and (9), then it can be expressed by

$u(n)$

$$
=\left\{\begin{array}{c}
\sum_{m=1}^{n} \phi_{q}\left(\sum_{i=m}^{n_{1}} \lambda a(i) f\left(i, u\left(i-n_{0}\right)\right)+\omega\right), \\
n \in \mathbf{Z}\left(1, n_{1}\right), \\
\sum_{m=n+1}^{N+1} \phi_{q}\left(\sum_{i=n_{1}+1}^{m-1} \lambda a(i) f\left(i, u\left(i-n_{0}\right)\right)-\omega\right), \\
n \in \mathbf{Z}\left(n_{1}+1, N\right), \\
n \in \mathbf{Z}\left(-n_{0}, 0\right), n=N+1,
\end{array}\right.
$$

where $\omega \in\left[0, \lambda a\left(n_{1}+1\right) f\left(n_{1}+1, u\left(n_{1}+1-n_{0}\right)\right)\right)$.

Let $C\left(\mathbf{Z}\left(-n_{0}, N+1\right), \mathbb{R}\right)$ denote the class of maps $u$ continuous on $\mathbf{Z}\left(-n_{0}, N+1\right)$ (discrete topology). We introduce a function space $E$, namely,

$$
\begin{gathered}
E=\left\{u \in C\left(\mathbf{Z}\left(-n_{0}, N+1\right), \mathbf{R}\right), u(N+1)\right. \\
\left.=0 ; u(n)=0, n \in \mathbf{Z}\left(-n_{0}, 0\right)\right\}
\end{gathered}
$$


with norm $\|\cdot\|$ given by $\|u\|=\max _{n \in \mathbf{Z}\left(-n_{0}, N+1\right)}|u(n)|$. Then $(E,\|\cdot\|)$ is a Banach space. Define a cone $K \subset E$ as follows:

$$
K=\{u \in E: u(n) \geq \mu(n)\|u\|, n \in \mathbf{Z}(0, N+1)\},
$$

where $\mu(n)=\min \{(N+1-n) /(N+1), n / N\}$.

From the definition of $\mu(n)$, we have

$$
\begin{aligned}
\mu(n) & =\min \left\{\frac{N+1-n}{N+1}, \frac{n}{N}\right\} \\
& = \begin{cases}\frac{n}{N}, & n \in \mathbf{Z}\left(1,\left[\frac{N}{2}\right]\right), \\
\frac{N+1-n}{N+1}, & n \in \mathbf{Z}\left(\left[\frac{N}{2}\right]+1, N\right) .\end{cases}
\end{aligned}
$$

Moreover, for any $u \in K$, we have $u(n) \geq(1 /(N+1))\|u\|$, $n \in \mathbf{Z}(1, N)$.

For any $u \in K$, define an operator $T$ on $K$ by

$(T u)(n)$

$$
=\left\{\begin{array}{c}
\sum_{m=1}^{n} \phi_{q}\left(\sum_{i=m}^{n_{1}} \lambda a(i) f\left(i, u\left(i-n_{0}\right)\right)+\omega\right), \\
n \in \mathbf{Z}\left(1, n_{1}\right), \\
\sum_{m=n+1}^{N+1} \phi_{q}\left(\sum_{i=n_{1}+1}^{m-1} \lambda a(i) f\left(i, u\left(i-n_{0}\right)\right)-\omega\right), \\
n \in \mathbf{Z}\left(n_{1}+1, N\right), \\
n \in \mathbf{Z}\left(-n_{0}, 0\right), n=N+1,
\end{array}\right.
$$

where $\omega \in\left[0, \lambda a\left(n_{1}+1\right) f\left(n_{1}+1, u\left(n_{1}+1-n_{0}\right)\right)\right)$.

Lemma 5. Suppose that (H1) and (H2) hold. Then the operator $T: K \rightarrow K$ is completely continuous.

Proof. For any $u \in K$, it is easily to check

$$
\begin{aligned}
\Delta\left(\phi_{p}(\Delta(T u)(n-1))\right) \\
=\phi_{p}((T u)(n+1)-(T u)(n)) \\
\quad-\phi_{p}((T u)(n)-(T u)(n-1)) \\
=-\lambda a(n) f\left(n, u\left(n-n_{0}\right)\right) \leq 0 .
\end{aligned}
$$

According to Lemma 3.2 in [1], we have $(T u)(n) \geq$ $\mu(n)\|T u\|$. Thus, $T(K) \subset K$. Moreover, $\phi_{p}$ and $f$ are both continuous, and $E$ is a finite space. This implies that the operator $T$ is a completely continuous. This completes the proof.
For convenience, we introduce some notations:

$$
\begin{aligned}
f_{\infty} & =\liminf _{u \rightarrow \infty} \min _{n \in \mathbf{Z}(1, N+1)} \frac{f(n, u)}{u^{p-1}}, \\
f^{0} & =\limsup _{u \rightarrow 0^{+}} \max _{n \in \mathbf{Z}(1, N+1)} \frac{f(n, u)}{u^{p-1}}, \\
f_{0} & =\liminf _{u \rightarrow 0^{+}} \min _{n \in \mathbf{Z}(1, N+1)} \frac{f(n, u)}{u^{p-1}}, \\
f^{\infty} & =\limsup _{u \rightarrow \infty} \max _{n \in \mathbf{Z}(1, N+1)} \frac{f(n, u)}{u^{p-1}} .
\end{aligned}
$$

If $u$ is a positive solution of problems (8) and (9), then it is a fixed point of $T$. So the existence of positive solution is transformed into the existence of fixed point. The following theorem is the main result of this paper.

Theorem 6. Suppose that (A1) and (A2) hold. Assume that the delay time is appropriately small, say $n_{0}$ is a positive integer not bigger than $[N / 2]$. If $f_{\infty}>0, f^{0}<\infty$, then there exists at least one positive solution to the boundary value problems (8) and (9) for $\lambda \in\left(\max \left\{a_{1}, a_{2}\right\}, b\right)$, where

$$
a_{1}=\frac{1}{f_{\infty} \cdot\left(\sum_{m=2}^{[N / 2]} \phi_{q}\left(\sum_{i=m}^{[N / 2]} a\left(i+n_{0}\right)(\mu(i))^{p-1}\right)\right)^{p-1}},
$$

$a_{2}$

$$
=\frac{1}{f_{\infty} \cdot\left(\sum_{m=2+[N / 2]}^{N+1-n_{0}} \phi_{q}\left(\sum_{i=1+[N / 2]}^{m-1} a\left(i+n_{0}\right)\left((\mu(i))^{p-1}\right)\right)^{p-1}\right.},
$$

$$
b=\frac{1}{f^{0} \cdot\left((N+1) \phi_{q}\left(\sum_{i=1}^{N+1} a(i)\right)\right)^{p-1}}
$$

while if $f_{0}>0, f^{\infty}<\infty$, then for any $\lambda \in\left(\max \left\{c_{1}, c_{2}\right\}, d\right)$, where

$$
\begin{aligned}
& c_{1}=\frac{1}{f_{0} \cdot\left(\sum_{m=2}^{[N / 2]} \phi_{q}\left(\sum_{i=m}^{[N / 2]} a\left(i+n_{0}\right)(\mu(i))^{p-1}\right)\right)^{p-1}}, \\
& c_{2} \\
& =\frac{1}{f_{0} \cdot\left(\sum_{m=2+[N / 2]}^{N+1-n_{0}} \phi_{q}\left(\sum_{i=1+[N / 2]}^{m-1} a\left(i+n_{0}\right)(\mu(i))^{p-1}\right)\right)^{p-1}}, \\
& d=\frac{1}{f^{\infty} \cdot\left((N+1) \phi_{q}\left(\sum_{i=1}^{N+1} a(i)\right)\right)^{p-1}},
\end{aligned}
$$

problems (8) and (9) admit at least one positive solution $u \in K$.

Proof. From Lemma 5, we see that $T: K \rightarrow K$ is a completely continuous operator. Since $\lambda \in\left(\max \left\{a_{1}, a_{2}\right\}, b\right)$, there exists an $\epsilon>0$ such that

$$
\max \left\{a_{1 \epsilon}, a_{2 \epsilon}\right\} \leq \lambda \leq b_{\epsilon},
$$


where

$$
\begin{aligned}
& a_{1 \epsilon} \\
& =\frac{1}{\left(f_{\infty}-\epsilon\right) \cdot\left(\sum_{m=2}^{[N / 2]} \phi_{q}\left(\sum_{i=m}^{[N / 2]} a\left(i+n_{0}\right)(\mu(i))^{p-1}\right)\right)^{p-1}}, \\
& a_{2 \epsilon} \\
& =\frac{1}{\left(f_{\infty}-\epsilon\right) \cdot\left(\sum_{m=2+[N / 2]}^{N+1-n_{0}} \phi_{q}\left(\sum_{i=1+[N / 2]}^{m-1} a\left(i+n_{0}\right)(\mu(i))^{p-1}\right)\right)^{p-1}}, \\
& \quad b_{\epsilon}=\frac{1}{\left(f^{0}+\epsilon\right) \cdot\left((N+1) \phi_{q}\left(\sum_{i=1}^{N+1} a(i)\right)\right)^{p-1}} .
\end{aligned}
$$

Let $\epsilon$ be fixed. By $f^{0}<\infty$, there exists an $M_{1}>0$ such that for $0 \leq u \leq M_{1}$,

$$
f(i, u) \leq\left(f^{0}+\epsilon\right) u^{p-1}, \quad i \in \mathbf{Z}(1, N+1) .
$$

Let $\Omega_{1}=\left\{u \in E:\|u\|<M_{1}\right\}$. Then for any $u \in K \cap \partial \Omega_{1}$, we have

$$
f\left(i, u\left(i-n_{0}\right)\right) \leq\left(f^{0}+\epsilon\right)\left(u\left(i-n_{0}\right)\right)^{p-1}, \quad i \in \mathbf{Z}(1, N+1) .
$$

In view of Lemma 1 and the definition of operator $T$, we further have

$$
\|T u\|=\max \left\{(T u)\left(n_{1}\right),(T u)\left(n_{1}+1\right)\right\} .
$$

Thus,

$\|T u\|$

$$
=\max \left\{(T u)\left(n_{1}\right),(T u)\left(n_{1}+1\right)\right\}
$$$$
=\max \left\{\sum_{m=1}^{n_{1}} \phi_{q}\left(\sum_{i=m}^{n_{1}} \lambda a(i) f\left(i, u\left(i-n_{0}\right)\right)+\omega\right),\right.
$$$$
\left.\sum_{m=n_{1}+2}^{N+1} \phi_{q}\left(\sum_{i=n_{1}+1}^{m-1} \lambda a(i) f\left(i, u\left(i-n_{0}\right)\right)-\omega\right)\right\}
$$$$
\leq \max \left\{\sum_{m=1}^{n_{1}} \phi_{q}\left(\sum_{i=m}^{n_{1}+1} \lambda a(i) f\left(i, u\left(i-n_{0}\right)\right)\right),\right.
$$

$$
\left.\sum_{m=n_{1}+2}^{N+1} \phi_{q}\left(\sum_{i=n_{1}+1}^{m-1} \lambda a(i) f\left(i, u\left(i-n_{0}\right)\right)\right)\right\}
$$

$$
\leq \max \left\{\sum_{m=1}^{n_{1}} \phi_{q}\left(\sum_{i=m}^{n_{1}+1} \lambda a(i)\left(f^{0}+\epsilon\right)\left(u\left(i-n_{0}\right)\right)^{p-1}\right),\right.
$$

$$
\left.\sum_{m=n_{1}+2}^{N+1} \phi_{q}\left(\sum_{i=n_{1}+1}^{m-1} \lambda a(i)\left(f^{0}+\epsilon\right)\left(u\left(i-n_{0}\right)\right)^{p-1}\right)\right\}
$$

$$
\begin{array}{r}
\leq \max \left\{\left(\lambda\left(f^{0}+\epsilon\right)\right)^{1 /(p-1)}\|u\| \sum_{m=1}^{n_{1}} \phi_{q}\left(\sum_{i=m}^{n_{1}+1} a(i)\right),\right. \\
\left.\left(\lambda\left(f^{0}+\epsilon\right)\right)^{1 /(p-1)}\|u\| \sum_{m=n_{1}+2}^{N+1} \phi_{q}\left(\sum_{i=n_{1}+1}^{m-1} a(i)\right)\right\} \\
\leq\left(\lambda\left(f^{0}+\epsilon\right)\right)^{1 /(p-1)}\|u\|(N+1) \phi_{q}\left(\sum_{i=1}^{N+1} a(i)\right) \leq\|u\| .
\end{array}
$$

Next, by $f_{\infty}>0$, it is easy to see that there exists an $\widetilde{M_{2}}>$ 0 such that for all $u>\widetilde{M_{2}}$,

$$
f(i, u) \geq\left(f_{\infty}-\epsilon\right) u^{p-1}, \quad i \in \mathbf{Z}(1, N+1) .
$$

Take $M_{2}=\max \left\{2 M_{1},(N+1) \widetilde{M_{2}}\right\}$. Let $\Omega_{2}=\{u \in E,\|u\|<$ $\left.M_{2}\right\}$. For any $u \in K \cap \partial \Omega_{2}$, we have

$$
\begin{array}{r}
f\left(i, u\left(i-n_{0}\right)\right) \geq\left(f_{\infty}-\epsilon\right)\left(u\left(i-n_{0}\right)\right)^{p-1}, \\
\forall i \in \mathbf{Z}\left(n_{0}+2, N+1\right) .
\end{array}
$$

Thus, if $n_{1}<n_{0}+[N / 2]$, we have

$\|T u\|$

$$
\begin{aligned}
& =\max \left\{(\mathrm{Tu})\left(n_{1}\right),(\mathrm{Tu})\left(n_{1}+1\right)\right\} \geq(\mathrm{Tu})\left(n_{1}+1\right) \\
& =\sum_{m=n_{1}+2}^{N+1} \phi_{q}\left(\sum_{i=n_{1}+1}^{m-1} \lambda a(i) f\left(i, u\left(i-n_{0}\right)\right)-\omega\right)
\end{aligned}
$$$$
\geq \sum_{m=n_{0}+[N / 2]+2}^{N+1} \phi_{q}\left(\sum_{i=n_{0}+[N / 2]+1}^{m-1} \lambda a(i) f\left(i, u\left(i-n_{0}\right)\right)\right)
$$$$
\geq \sum_{m=n_{0}+[N / 2]+2}^{N+1} \phi_{q}
$$$$
\times\left(\sum_{i=n_{0}+[N / 2]+1}^{m-1} \lambda a(i)\left(f_{\infty}-\epsilon\right)\left(u\left(i-n_{0}\right)\right)^{p-1}\right)
$$$$
\geq \sum_{m=[N / 2]+2}^{N+1-n_{0}} \phi_{q}\left(\sum_{i=[N / 2]+1}^{m-1} \lambda a\left(i+n_{0}\right)\left(f_{\infty}-\epsilon\right)(u(i))^{p-1}\right)
$$$$
\geq\|u\| \sum_{m=[N / 2]+2}^{N+1-n_{0}} \phi_{q}\left(\sum_{i=[N / 2]+1}^{m-1} \lambda a\left(i+n_{0}\right)\left(f_{\infty}-\epsilon\right)(\mu(i))^{p-1}\right)
$$$$
\geq\left(\lambda\left(f_{\infty}-\epsilon\right)\right)^{1 /(p-1)}\|u\|
$$$$
\times \sum_{m=[N / 2]+2}^{N+1-n_{0}} \phi_{q}\left(\sum_{i=[N / 2]+1}^{m-1} a\left(i+n_{0}\right)(\mu(i))^{p-1}\right) \geq\|u\| ;
$$ 
while if $n_{1} \geq n_{0}+[N / 2]$, then we have

$$
\begin{aligned}
\|T u\|= & \max \left\{(T u)\left(n_{1}\right),(T u)\left(n_{1}+1\right)\right\} \\
\geq & (T u)\left(n_{1}\right) \\
= & \sum_{m=1}^{n_{1}} \phi_{q}\left(\sum_{i=m}^{n_{1}} \lambda a(i) f\left(i, u\left(i-n_{0}\right)\right)+\omega\right) \\
\geq & \sum_{m=n_{0}+2}^{n_{0}+[N / 2]} \phi_{q}\left(\sum_{i=m}^{n_{0}+[N / 2]} \lambda a(i) f\left(i, u\left(i-n_{0}\right)\right)\right) \\
\geq & \sum_{m=2}^{[N / 2]} \phi_{q}\left(\sum_{i=m}^{[N / 2]} \lambda a\left(i+n_{0}\right)\left(f_{\infty}-\epsilon\right)(u(i))^{p-1}\right) \\
\geq & \left(\lambda\left(f_{\infty}-\epsilon\right)\right)^{1 /(p-1)}\|u\| \\
& \times \sum_{m=2}^{[N / 2]} \phi_{q}\left(\sum_{i=m}^{[N / 2]} a\left(i+n_{0}\right)(\mu(i))^{p-1}\right) \geq\|u\| .
\end{aligned}
$$

Combining the two estimates above, we deduce $\|T u\| \geq$ $\|u\|$, when $u \in K \cap \partial \Omega_{1}$. Therefore, by Theorem 3, $T$ has a fixed point $u \in K \cap\left(\bar{\Omega}_{2} \backslash \Omega_{1}\right)$. Namely, $u(t)$ is a positive solution of problems (8) and (9).

The proof of the second half is parallel to above. There exists an $\epsilon>0$ such that

$$
\max \left\{c_{1 \epsilon}, c_{2 \epsilon}\right\} \leq \lambda \leq d_{\epsilon}
$$

where

$$
\begin{gathered}
c_{1 \epsilon}=\frac{1}{\left(f_{0}-\epsilon\right) \cdot\left(\sum_{m=2}^{[N / 2]} \phi_{q}\left(\sum_{i=m}^{[N / 2]} a\left(i+n_{0}\right)(\mu(i))^{p-1}\right)\right)^{p-1}}, \\
c_{2 \epsilon} \\
=\frac{1}{\left(f_{0}-\epsilon\right) \cdot\left(\sum_{m=2+[N / 2]}^{N+1-n_{0}} \phi_{q}\left(\sum_{i=1+[N / 2]}^{m-1} a\left(i+n_{0}\right)(\mu(i))^{p-1}\right)\right)^{p-1}}, \\
d_{\epsilon}=\frac{1}{\left(f^{\infty}+\epsilon\right) \cdot\left((N+1) \phi_{q}\left(\sum_{i=1}^{N+1} a(i)\right)\right)^{p-1}} .
\end{gathered}
$$

Let $\epsilon$ be fixed. By $f_{0}>0$, then it is not difficult to choose an $M_{1}>0$ such that $0 \leq u \leq M_{1}$,

$$
f(i, u) \geq\left(f_{0}-\epsilon\right) u^{p-1}, \quad i \in \mathbf{Z}(1, N+1) .
$$

Let $\Omega_{1}=\left\{u \in E,\|u\|<M_{1}\right\}$. Then for $u \in K \cap \partial \Omega_{1}$, it can be obtained that

$f\left(i, u\left(i-n_{0}\right)\right) \geq\left(f_{0}-\epsilon\right)\left(u\left(i-n_{0}\right)\right)^{p-1}, \quad i \in \mathbf{Z}(1, N+1)$.
If $n_{1}<n_{0}+[N / 2]$, we have

$\|T u\|$

$$
\begin{aligned}
= & \max \left\{(T u)\left(n_{1}\right),(T u)\left(n_{1}+1\right)\right\} \\
\geq & (T u)\left(n_{1}+1\right) \\
= & \sum_{m=n_{1}+2}^{N+1} \phi_{q}\left(\sum_{i=n_{1}+1}^{m-1} \lambda a(i) f\left(i, u\left(i-n_{0}\right)\right)-\omega\right) \\
\geq & \sum_{m=n_{0}+[N / 2]+2}^{N+1} \phi_{q}\left(\sum_{i=n_{0}+[N / 2]+1}^{m-1} \lambda a(i) f\left(i, u\left(i-n_{0}\right)\right)\right) \\
\geq & \sum_{m=n_{0}+[N / 2]+2}^{N+1} \phi_{q}\left(\sum_{i=n_{0}+[N / 2]+1}^{m-1} \lambda a(i)\left(f_{0}-\epsilon\right)\left(u\left(i-n_{0}\right)\right)^{p-1}\right) \\
\geq & \|u\| \sum_{m=[N / 2]+2}^{N+1-n_{0}} \phi_{q}\left(\sum_{i=[N / 2]+1}^{m-1} \lambda a\left(i+n_{0}\right)\left(f_{0}-\epsilon\right)(\mu(i))^{p-1}\right) \\
\geq & \left(\lambda\left(f_{0}-\epsilon\right)\right)^{1 /(p-1)}\|u\| \\
& \times \sum_{m=[N / 2]+2}^{N+1-n_{0}} \phi_{q}\left(\sum_{i=[N / 2]+1}^{m-1} a\left(i+n_{0}\right)(\mu(i))^{p-1}\right) \geq\|u\| .
\end{aligned}
$$

Conversely, if $n_{1} \geq n_{0}+[N / 2]$, then we have

\section{$\|T u\|$}

$$
\begin{aligned}
& =\max \left\{(T u)\left(n_{1}\right),(\mathrm{Tu})\left(n_{1}+1\right)\right\} \\
& \geq(T u)\left(n_{1}\right) \\
& =\sum_{m=1}^{n_{1}} \phi_{q}\left(\sum_{i=m}^{n_{1}} \lambda a(i) f\left(i, u\left(i-n_{0}\right)\right)+\omega\right) \\
& \geq \sum_{m=n_{0}+2}^{n_{0}+[N / 2]} \phi_{q}\left(\sum_{i=m}^{n_{0}+[N / 2]} \lambda a(i) f\left(i, u\left(i-n_{0}\right)\right)\right) \\
& \geq \sum_{m=2}^{[N / 2]} \phi_{q}\left(\sum_{i=m}^{[N / 2]} \lambda a\left(i+n_{0}\right)\left(f_{0}-\epsilon\right)(u(i))^{p-1}\right) \\
& \geq\left(\lambda\left(f_{0}-\epsilon\right)\right)^{1 /(p-1)}\|u\| \sum_{m=2}^{[N / 2]} \phi_{q}\left(\sum_{i=m}^{[N / 2]} a\left(i+n_{0}\right)(\mu(i))^{p-1}\right) \\
& \geq\|u\| .
\end{aligned}
$$
$\widetilde{M_{2}}$

Next, by $f^{\infty}<\infty$, there exists $\widetilde{M_{2}}>0$ such that for $u>$

$$
f(i, u) \leq\left(f^{\infty}+\epsilon\right) u^{p-1}, \quad i \in \mathbf{Z}(1, N+1) .
$$


If $f$ is bounded, then there exists an $N_{1}>0$ such that $f(i, u) \leq N_{1}$ for $i \in \mathbf{Z}(1, N+1), 0 \leq u<\infty$. Take $M_{2}=$ $\max \left\{2 M_{1},\left(\lambda N_{1}\right)^{1 /(p-1)}(N+1) \phi_{q}\left(\sum_{i=1}^{N+1} a(i)\right)\right\}$ and $\Omega_{2}=\{u \in$ $\left.E,\|u\|<M_{2}\right\}$. Then for any $u \in K \cap \partial \Omega_{2}$, we have

\section{$\|T u\|$}

$$
\begin{aligned}
& =\max \left\{(T u)\left(n_{1}\right),(T u)\left(n_{1}+1\right)\right\} \\
& =\max \left\{\sum_{m=1}^{n_{1}} \phi_{q}\left(\sum_{i=m}^{n_{1}} \lambda a(i) f\left(i, u\left(i-n_{0}\right)\right)+\omega\right),\right. \\
& \leq \max \left\{\sum_{m=n_{1}+2}^{n_{1}} \phi_{q}\left(\sum_{i=n_{1}+1}^{n_{1}} \lambda a(i) f\left(i, u\left(i-n_{0}\right)\right)-\omega\right)\right\} \\
& \leq \max \left\{\sum_{m=1}^{n_{1}+1} \phi_{q}\left(\sum_{i=m}^{n_{1}} \lambda a(i) f\left(i, u\left(i-n_{0}\right)\right)\right),\right. \\
& \left.\sum_{m=n_{1}+2}^{n_{1}+1} \phi_{q}\left(\sum_{i=n_{1}+1}^{m-1} \lambda a(i) f\left(i, u\left(i-n_{0}\right)\right)\right)\right\} \\
& \leq(N+1)\left(\lambda N_{1}\right)^{1 /(p-1)} \phi_{q}\left(\sum_{i=1}^{N+1} a(i)\right) \leq M_{2}=\|u\| .
\end{aligned}
$$

While if $f$ is unbounded, it is not difficult to see that there exists an $M_{2}>\max \left\{2 M_{1}, \widetilde{M_{2}}\right\}, i_{0} \in \mathbf{Z}(1, N)$ such that $0 \leq u \leq$ $M_{2}, f(i, u) \leq f\left(i_{0}, M_{2}\right)$. Take $\Omega_{2}=\left\{u \in E:\|u\|<M_{2}\right\}$, then for $u \in K \cap \partial \Omega_{2}$, we get

$$
\begin{aligned}
& \|T u\| \\
& =\max \left\{(T u)\left(n_{1}\right),(T u)\left(n_{1}+1\right)\right\} \\
& =\max \left\{\sum_{m=1}^{n_{1}} \phi_{q}\left(\sum_{i=m}^{n_{1}} \lambda a(i) f\left(i, u\left(i-n_{0}\right)\right)+\omega\right),\right. \\
& \leq \max \left\{\sum_{m=1}^{N+1} \phi_{q}\left(\sum_{i=m}^{n_{1}} \lambda\left(\sum_{i=n_{1}+1}^{m-1} \lambda a(i) f\left(i, u\left(i-n_{0}\right)\right)-\omega\right)\right\}\right. \\
& \left.\sum_{m=n_{1}+2}^{n_{1}+1} \phi_{q}\left(\sum_{i=n_{1}+1}^{m-1} \lambda a(i) f\left(i, u\left(i-n_{0}\right)\right)\right)\right\}
\end{aligned}
$$

$$
\begin{aligned}
& \leq \max \left\{\sum_{m=1}^{n_{1}} \phi_{q}\left(\sum_{i=m}^{n_{1}+1} \lambda a(i) f\left(i_{0}, M_{2}\right)\right),\right. \\
&\left.\sum_{m=n_{1}+2}^{N+1} \phi_{q}\left(\sum_{i=n_{1}+1}^{m-1} \lambda a(i) f\left(i_{0}, M_{2}\right)\right)\right\}
\end{aligned}
$$

$$
\begin{gathered}
\leq \max \left\{\left(\lambda\left(f^{\infty}+\epsilon\right)\right)^{1 /(p-1)} M_{2} \sum_{m=1}^{n_{1}} \phi_{q}\left(\sum_{i=m}^{n_{1}+1} a(i)\right),\right. \\
\left.\left(\lambda\left(f^{\infty}+\epsilon\right)\right)^{1 /(p-1)} M_{2} \sum_{m=n_{1}+2}^{N+1} \phi_{q}\left(\sum_{i=n_{1}+1}^{m-1} a(i)\right)\right\} \\
\leq(N+1)\left(\lambda\left(f^{\infty}+\epsilon\right)\right)^{1 /(p-1)} M_{2} \phi_{q}\left(\sum_{i=1}^{N+1} a(i)\right) \leq M_{2}=\|u\| .
\end{gathered}
$$

Therefore, by Theorem 3, $T$ has a fixed point $u \in K \cap\left(\bar{\Omega}_{2} \backslash\right.$ $\left.\Omega_{1}\right)$. Namely, $u$ is a positive solution of problema (8) and (9). This completes the proof.

\section{Numerical Illustration}

In this section, we present two numerical experiments to illustrate our results.

Example 7. Consider the following boundary value problem:

$$
\begin{array}{r}
u(n+1)-2 u(n)+u(n-1)+a(n) f(n, u(n-5))=0, \\
n \in \mathbf{Z}(1,99), \\
u(100)=0, \quad u(n)=0, \quad n \in \mathbf{Z}(-5,0),
\end{array}
$$

where $a(n)=n / 100, f(n, u(n-5))=(1 / 100)^{2}(u(n-5))^{2}$. It is easy to see that $f_{\infty}=+\infty, f^{0}=0$. By Theorem 6 , problem (56) has a positive solution. In Figure 1, we show the numerical solution to problem (56).

Example 8. Consider the following boundary value problem

$$
\begin{gathered}
\Delta \phi_{4}(\Delta u(n-1))+a(n) f(n, u(n-3))=0, \quad n \in \mathbf{Z}(1,99), \\
u(100)=0, \quad u(n)=0, \quad n \in \mathbf{Z}(-3,0),
\end{gathered}
$$

where $a(n) \equiv 1, f(n, u(n-3))=(1 / 100)^{4}(u(n-3))^{2}$. We check that $f^{\infty}=0, f_{0}=+\infty$. Then applying Theorem 6 , problem (57) has a positive solution. Numerically, we obtain the solution of problem (57) which is shown in Figure 1.

\section{Acknowledgments}

This work is supported by Tian Yuan Fund of China (11226308), NNSF of China (11071102), Natural Science Fund for Colleges and Universities in Jiangsu Province (11KJD110001), the Research Fund (10JDG124) for Highlevel 




(a) The numerical solution to problem (56)

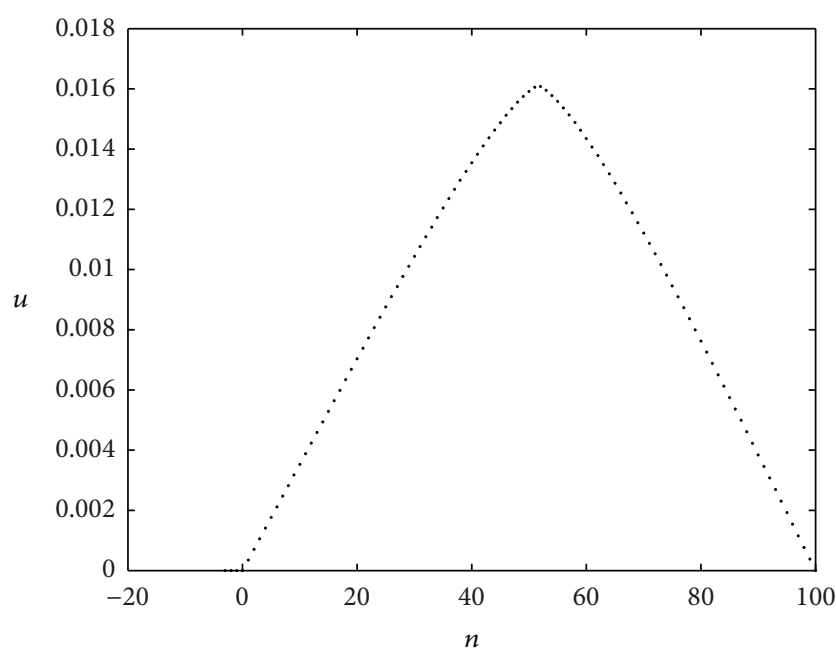

(b) The numerical solution to problem (57)

FIGURE 1: The figures of the numerical solutions.

Group of Jiangsu University, and Postdoctoral Science Foundation (2011M500874, 1002030C).

\section{References}

[1] D. Q. Jiang, J. F. Chu, D. O’Regan, and R. P. Agarwal, “Positive solutions for continuous and discrete boundary value problems to the one-dimension $p$-Laplacian," Mathematical Inequalities \& Applications, vol. 7, no. 4, pp. 523-534, 2004.

[2] W.-T. Li and H.-F. Huo, "Positive periodic solutions of delay difference equations and applications in population dynamics," Journal of Computational and Applied Mathematics, vol. 176, no. 2, pp. 357-369, 2005.

[3] Y. J. Liu, "A study on periodic solutions of higher order nonlinear functional difference equations with $p$-Laplacian," Journal of Difference Equations and Applications, vol. 13, no. 12, pp. 1105-1114, 2007.

[4] H. Y. Feng, W. G. Ge, and M. Jiang, "Multiple positive solutions for $m$-point boundary-value problems with a one-dimensional
p-Laplacian," Nonlinear Analysis. Theory, Methods \& Applications, vol. 68, no. 8, pp. 2269-2279, 2008.

[5] C.-G. Kim, "Existence of positive solutions for singular boundary value problems involving the one-dimensional p-Laplacian," Nonlinear Analysis. Theory, Methods \& Applications, vol. 70, no. 12, pp. 4259-4267, 2009.

[6] R. Glowinski and J. Rappaz, "Approximation of a nonlinear elliptic problem arising in a non-Newtonian fluid flow model in glaciology," Mathematical Modelling and Numerical Analysis, vol. 37, no. 1, pp. 175-186, 2003.

[7] C. H. Jin, J. X. Yin, and Z. J. Wang, "Positive radial solutions of $p$-Laplacian equation with sign changing nonlinear sources," Mathematical Methods in the Applied Sciences, vol. 30, no. 1, pp. 1-14, 2007.

[8] D. Y. Bai and Y. T. Xu, "Existence of positive solutions for boundary-value problems of second-order delay differential equations," Applied Mathematics Letters, vol. 18, no. 6, pp. 621630, 2005.

[9] C. H. Jin and J. X. Yin, "Positive solutions for the boundary value problems of one-dimensional $p$-Laplacian with delay," Journal of Mathematical Analysis and Applications, vol. 330, no. 2, pp. 1238-1248, 2007.

[10] L. Wei and J. Zhu, "The existence and blow-up rate of large solutions of one-dimensional $p$-Laplacian equations," Nonlinear Analysis. Real World Applications, vol. 13, no. 2, pp. 665-676, 2012.

[11] Z. L. Yang and D. O’Regan, "Positive solutions of a focal problem for one-dimensional $p$-Laplacian equations," Mathematical and Computer Modelling, vol. 55, no. 7-8, pp. 1942-1950, 2012.

[12] Z. M. He, "On the existence of positive solutions of $p$-Laplacian difference equations," Journal of Computational and Applied Mathematics, vol. 161, no. 1, pp. 193-201, 2003.

[13] R. I. Avery and J. Henderson, "Existence of three positive pseudo-symmetric solutions for a one-dimensional $p$ Laplacian," Journal of Mathematical Analysis and Applications, vol. 277, no. 2, pp. 395-404, 2003.

[14] D.-B. Wang and W. Guan, "Three positive solutions of boundary value problems for $p$-Laplacian difference equations," Computers \& Mathematics with Applications, vol. 55, no. 9, pp. 19431949, 2008.

[15] P. Candito and N. Giovannelli, "Multiple solutions for a discrete boundary value problem involving the $p$-Laplacian," Computers \& Mathematics with Applications, vol. 56, no. 4, pp. 959-964, 2008.

[16] A. Iannizzotto and S. A. Tersian, "Multiple homoclinic solutions for the discrete $p$-Laplacian via critical point theory," Journal of Mathematical Analysis and Applications, vol. 403, no. 1, pp. 173182, 2013.

[17] Y. J. Liu and X. Y. Liu, "The existence of periodic solutions of higher order nonlinear periodic difference equations," Mathematical Methods in the Applied Sciences, vol. 36, pp. 1459-1470, 2013.

[18] H. H. Liang and P. X. Weng, "Existence and multiple solutions for a second-order difference boundary value problem via critical point theory," Journal of Mathematical Analysis and Applications, vol. 326, no. 1, pp. 511-520, 2007.

[19] D. J. Guo and V. Lakshmikantham, Nonlinear Problems in Abstract Cones, vol. 5, Academic Press, Boston, Mass, USA, 1988. 


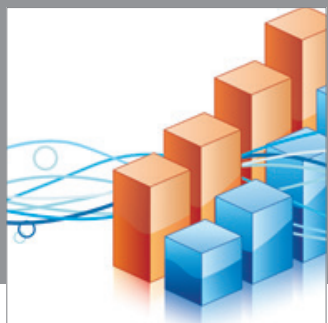

Advances in

Operations Research

mansans

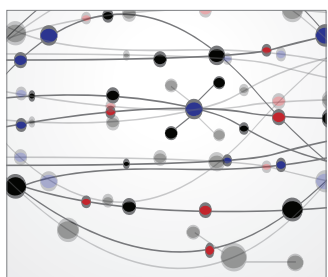

The Scientific World Journal

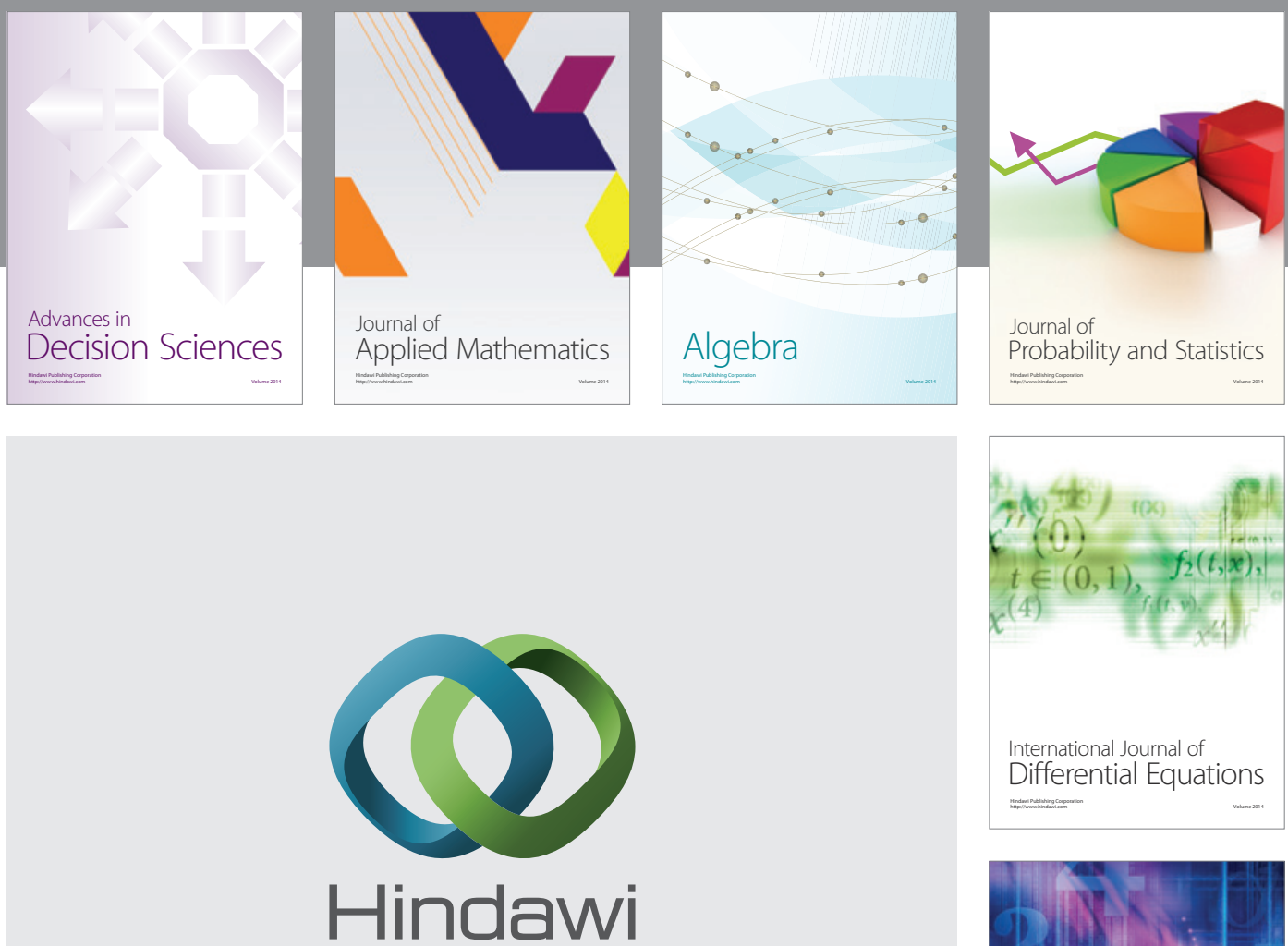

Submit your manuscripts at http://www.hindawi.com


Journal of

Function Spaces

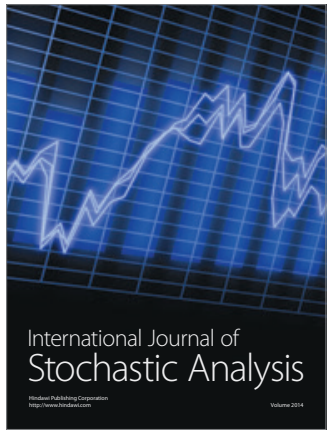

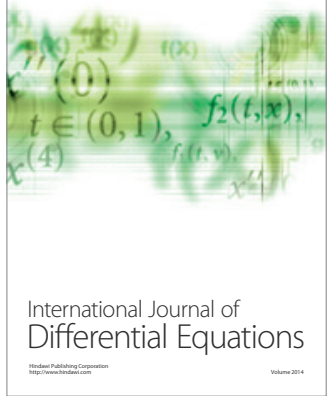
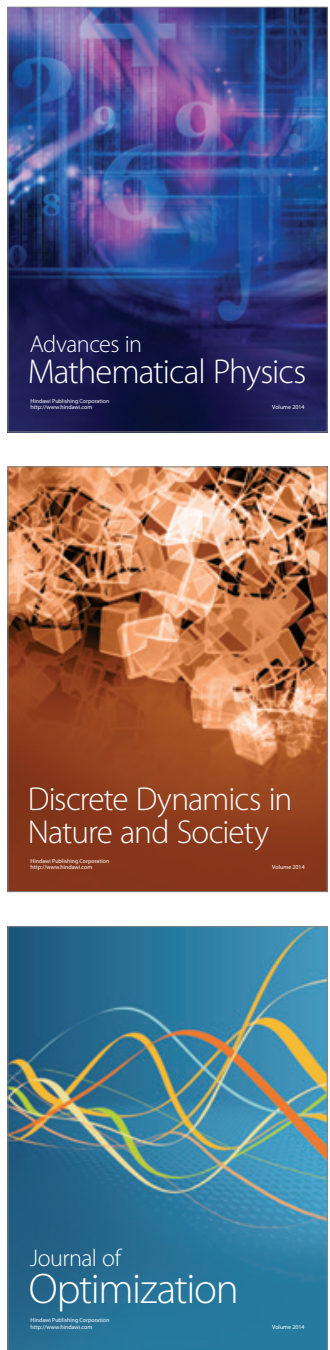